\title{
UK Renal Registry 16th Annual Report: Appendix J Laboratory Conversion Factors
}

\begin{tabular}{ll}
\hline & Conversion factors from SI units \\
\hline Albumin & $\mathrm{g} / \mathrm{dl}=\mathrm{g} / \mathrm{L} \times 0.1$ \\
Aluminium & $\mu \mathrm{g} / \mathrm{L}=\mu \mathrm{mol} / \mathrm{L} \times 27.3$ \\
Bicarbonate & $\mathrm{mg} / \mathrm{dl}=\mathrm{mmol} / \mathrm{L} \times 6.1$ \\
Calcium & $\mathrm{mg} / \mathrm{dl}=\mathrm{mmol} / \mathrm{L} \times 4$ \\
Calcium $\times$ phosphate & $\mathrm{mg} / \mathrm{dl}^{2}=\mathrm{mmol} / \mathrm{L}^{2} \times 12.4$ \\
Cholesterol & $\mathrm{mg} / \mathrm{dl}=\mathrm{mmol} / \mathrm{L} \times 38.6$ \\
Creatinine & $\mathrm{mg} / \mathrm{dl}=\mu \mathrm{mol} / \mathrm{L} \times 0.011$ \\
Glucose & $\mathrm{mg} / \mathrm{d}=\mathrm{mmol} / \mathrm{L} \times 18$ \\
Phosphate & $\mathrm{mg} / \mathrm{dl}=\mathrm{mmol} / \mathrm{L} \times 3.1$ \\
PTH & $\mathrm{ng} / \mathrm{L}=\mathrm{pmol} / \mathrm{L} \times 9.5$ \\
Urea & $\mathrm{mg} / \mathrm{dl}=\mathrm{mmol} / \mathrm{L} \times 6.0$ \\
Urea nitrogen & $\mathrm{mg} / \mathrm{dl}=\mathrm{mmol} / \mathrm{L} \times 2.8$ \\
\hline
\end{tabular}

\section{KARGER}

Fax +4161306 1234

E-Mail karger@karger.com www.karger.com
UK Renal Registry, Southmead Hospital, Southmead Road, Bristol, BS10 5NB, UK

Email: renalregistry@renalregistry.nhs.uk 